\title{
Evaluation of Glycemia Control Achieved by Glargine and Lispro Versus Detemir and Aspart Insulin Regimes in Type 2 Diabetics Undergoing Surgery
}

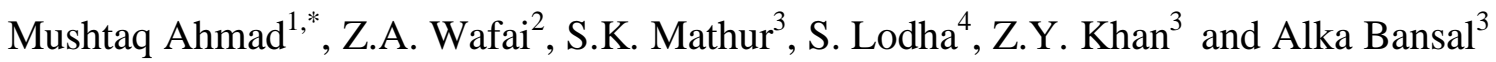 \\ ${ }^{1}$ Department of Pharmacology, Punjab Institute of Medical Sciences, Jalandhar \\ ${ }^{2}$ Department of Clinical Pharmacology, Sheri-I- Kashmir Institute of Medical Sciences, Srinagar \\ ${ }^{3}$ Department of Endocrinology, S. M. S. Medical College \& Hospital, Jaipur \\ ${ }^{4}$ Department of Endocrinology, Fortis Escorts Hospital, Jaipur
}

\begin{abstract}
There is paucity of scientific literature regarding the clinical outcome of long lasting basal insulin and rapid acting mealtime insulin regimes in surgical situations although employed in non-surgical situations. This study has evaluated the clinical outcome of two subcutaneous split-mixed Glargine+Lispro and Detemir+Aspart insulin regimes in type 2 diabetics undergoing surgery.
\end{abstract}

Keywords: Aspart, Detemir, Glargine, Lispro, Surgery, Type 2 DM patients.

\section{INTRODUCTION}

Diabetes mellitus (DM) is a metabolic disorder characterized by relative or absolute deficiency of insulin. It is an established fact that the patients with diabetes undergo surgery at a higher rate than non-diabetics and are prone to various adverse outcomes [1]. Surgery invokes a neuroendocrine stress response with release of counter regulatory hormones resulting in peripheral insulin resistance, increased hepatic glucose production, impaired insulin secretion and fat and protein breakdown with potential hyperglycemia and even ketosis in some cases [2]. The degree of this response depends on the complexity of the surgery and any postsurgical complications. To reduce the aggravation of the untoward effects of concomitant illness and morbidity the optimal glycaemia management during surgery is desirable [3].

Subcutaneous insulin therapy is a common practice in replacement of oral drug therapy to control peri-operative hyperglycaemia. The combination of a long acting basal and rapid acting meal time insulin has been recommended as a more physiological approach to glucose control in hospitalized diabetics $[4,5]$. Though several studies have compared long acting insulin with NPH in non-surgical situations but there is paucity of scientific literature regarding use of basal bolus insulin regimes in surgical situations. Many insulin and oral hypoglycemic treatment regimes depending upon the type of diabetes, nature

*Address correspondence to this author at the Punjab Institute of Medical Sciences, Punjab, India; Tel: +91-8699013755;

E-mail: marumush@gmail.com of surgery and timing of operation have been recommended for glycemia control during peri-operative period [6, 7]. However, the literature regarding their superiority of glycemia control in terms of surgical outcome during perioperative period is insufficient. Hence, this study was planned to evaluate two different subcutaneous insulin regimes in terms of glycemia control and the incidence of complications in type 2 DM patients undergoing surgery.

\section{MATERIALS AND METHODOLOGY}

140 type 2 DM patients admitted to surgical wards to undergo major elective surgery were enrolled. All the patients were referred to endocrinology unit of the participating hospital for metabolic management. The patients in whom the preoperative hyperglycemia was to be controlled with subcutaneous insulin were studied. Old and newly diagnosed type $2 \mathrm{DM}$ patients of age between 18-70 years were included. The patients on concomitant use of oral antidiabetics, chronic corticosteroid or any other drug that either precipitates or aggravates the DM were excluded. In addition, the patients undergoing coronary artery bypass grafting surgery were also excluded. Written, informed consent of all the patients and approval of Institutional Ethics Committee (IEC) was obtained before starting the study.

\section{Study Design and Protocol}

This was a multi-centric, prospective study conducted at M. G. Hospital, S.M.S. Hospital and Fortis Escorts Hospital, 
Jaipur, India. On enrolling the patients, routine investigation of fasting, random and post prandial blood glucose was done twice for confirmation. After educating the participating patients on diet, importance of insulin with special emphasis on need to adhere to treatment, they were randomly allocated to two treatment groups A and B respectively receiving splitmixed Glargine+Lispro and split-mixed Detemir+Aspart. A simple randomization technique was followed for treatment allocation. The insulin preparation was kept under the control of the ward nurse who actually was administering the respective dose of the insulin. Each patient was given multiple injections into the subcutaneous tissue of the upper arm, anterior and lateral aspects of the thigh, buttocks and abdomen preoperatively till a day before the surgery and a day after the surgery until the patients were switched back to the same treatment regime they received preoperatively. The starting dose of the insulin was 0.5 units per kg body weight. Insulin dose was adjusted according to the pre- and postmeal blood glucose values. Any increase or decrease in insulin requirement was noted and compared with the goal to maintain the average blood glucose level between 120-180 $\mathrm{mg} / \mathrm{dl}$. In both the split-mixed regimes, ultra-short acting insulin was given subcutaneously in three equally divided doses with each meal and long acting insulin was administered regardless of the patient's oral intake status in the evening at bed time.

\section{STATISTICAL ANALYSIS}

Chi-square and ANOVA (one way classification) tests were employed for statistical analysis. The data was analyzed with SPSS software (Version 10.0). A probability value of less than $0.05(\mathrm{p}<0.05)$ was considered to be statistically significant.

\section{RESULTS}

A total of 140 type 2 diabetes mellitus patients who were allocated to two treatment groups viz A and B received split- mixed Glargine+Lispro and Detemir+Aspart subcutaneous insulin regimes, respectively. Both the treatment groups had more or less similar clinical and demographic characteristics. The most common admitting outcomes assessed were glycemia control, hypoglycemic episodes and postoperative complications.

Mean fasting blood glucose obtained a day before starting the treatment has significantly reduced $(\mathrm{p}<0.001)$ when compared to mean blood glucose levels achieved in both the regimes A $(233 \pm 67)$ and B $(227 \pm 63)$ a day prior to surgery (Fig. 1). While assessing the glycemia control a statistically significant difference existed in before breakfast $(p<0.05)$ and before dinner $(p<0.01)$ mean blood glucose levels between groups preoperatively. However, there was no intragroup or intergroup significant difference $(p>0.05)$ in other mean preoperative or postoperative blood glucose levels though the levels were approximately within a target range of 120-180 mg/dl before and after surgery (Fig. 1).

$13.0 \%$ hypoglycemic episodes were recorded in patients who were treated with Glargine+Lispro insulin regime while as $15.1 \%$ hypoglycemic episodes were recorded in patients who were treated with Detemir+Aspart insulin regime. However, this difference in incidence of hypoglycemic episodes between the regimes was statistically insignificant (p>0.05) (Fig. 2). Comparing the incidence of postoperative complications, $11.59 \%$ wound infections were recorded in treatment group A and $7.04 \%$ wound infections in treatment group B though this difference was statistically insignificant (p>0.05). However, a significant difference existed in gastrointestinal complications $(\mathrm{p}<0.001)$ and urinary tract infections $(p<0.05)$ as no incidence of urinary tract infection was observed in group A and no incidence of gastrointestinal complication was observed in group B. Interestingly, the incidence of total number of complications recorded in group $\mathrm{B}$ were significantly lower $(\mathrm{p}<0.05)$ than group A. The mean daily insulin dose to achieve the glycemic target was $30.46 \pm 1.4$ units and $31.5 \pm 0.6$ units in regime $\mathrm{A}$ and $\mathrm{B}$ respectively.

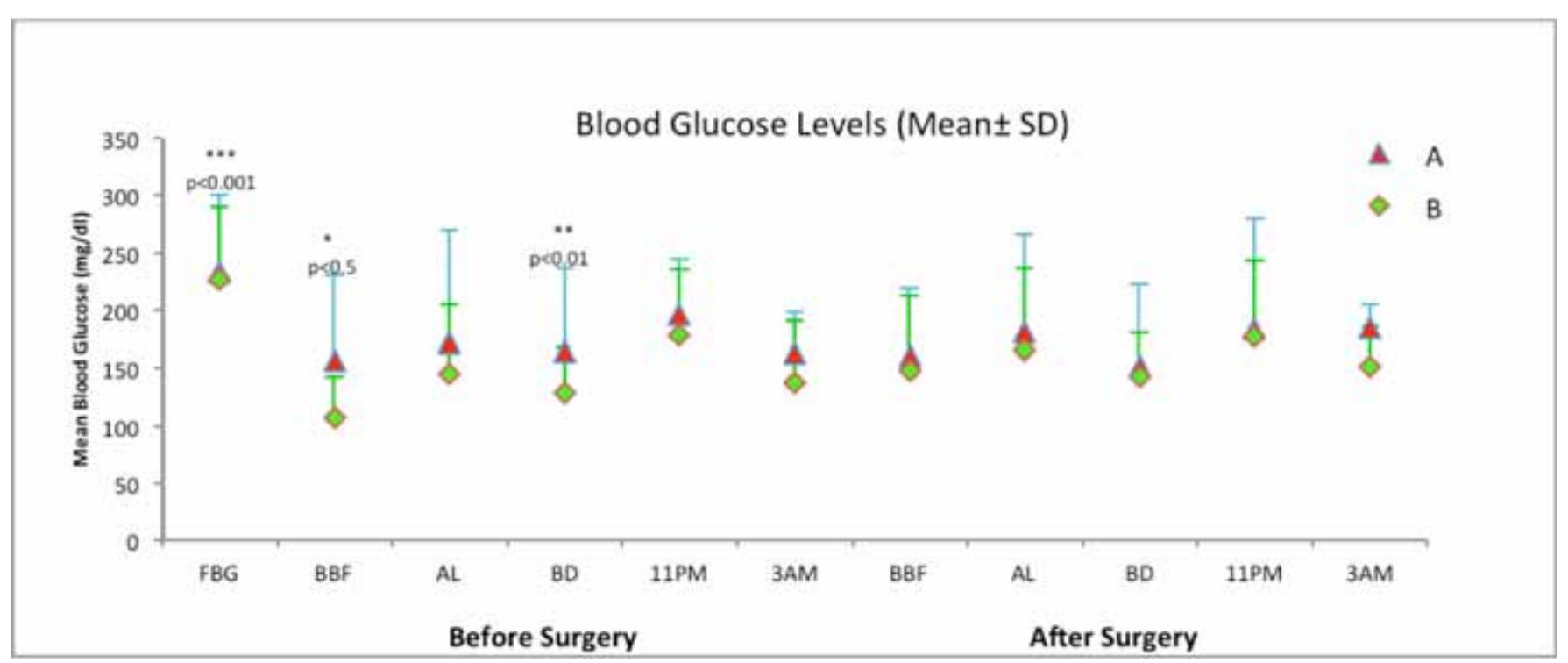

Fig. (1). Comparison of mean blood glucose levels before and after surgery. FBG=Fasting blood glucose before treatment, BBF: Before breakfast, $\mathrm{AL}=$ After lunch, $\mathrm{BD}=$ Before dinner, $\mathrm{A}=$ Glargine + Lispro (split-mixed), $\mathrm{B}=$ Detemir + Aspart (split-mixed). 


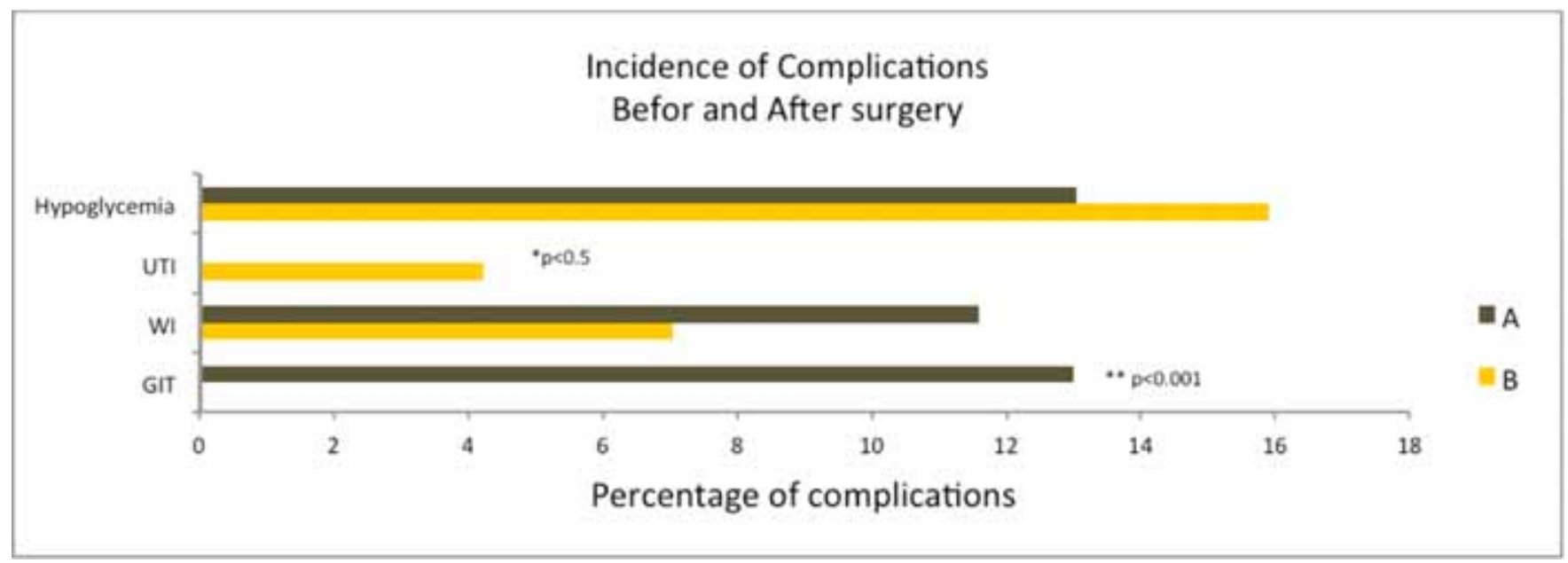

Fig. (2). Incidence of complications before and after surgery. UTI=urinary tract infection, WI=wound infection, GIT=gastrointestinal complications, A= Glargine + Lispro (split-mixed), B= Detemir + Aspart (split-mixed).

\section{DISCUSSION}

It is an established fact that a known diabetic patient has $50 \%$ risk of undergoing surgery during his lifetime and nearly one-fifth of the surgical patients have diabetes [8]. Diabetes is associated with increased requirement for surgical procedures and increased postoperative morbidity and mortality [9]. Hyperglycemia inhibits host defenses against infection [10-12] and impairs wound healing because of its detrimental effects on collagen formation resulting in diminished wound tensile strength [13, 14]. Various scientific studies have made it clear now that the norepinephrine released during surgery and epinephrine released postoperatively favour hyperglycemia, lipolysis and ketogenesis [8]. These aforementioned changes when severe are responsible for the development of hyperosmolar coma and uncontrolled growth of microorganisms if not interfered in time. The aim of therapy for hyperglycemia in diabetics undergoing surgery is not to treat the diabetes per se but to maintain and control the blood glucose level within a target range so as to achieve the satisfactory, timely and rapid recovery from surgical stress with minimum possible perioperative complications.

Various insulin regimes are employed for metabolic control in diabetics undergoing surgery. It is hypothesized that the combination of long acting basal and rapid acting meal time insulin is more physiological $[4,5]$. Hence, it was found worthwhile to evaluate the combination of two long acting basal and rapid acting meal time subcutaneous insulin regimes for glycemia control, incidence of hypoglycemic episodes and postoperative complications in type $2 \mathrm{DM}$ patients undergoing surgery. The mean fasting blood glucose recorded a day before starting the treatment in both the groups was significantly reduced $(\mathrm{p}<0.001)$ when compared to mean blood glucose levels recorded a day prior to surgery (Fig. 1). Interestingly while assessing the glycemia control a difference was recorded in mean blood glucose levels pre and postoperatively between the treatment regimes. However, this difference was not statistically significant ( $p>0.05$ ) except for before breakfast and before dinner mean blood glucose levels recorded preoperatively though the levels were approximately within a target range of 120-180 $\mathrm{mg} / \mathrm{dl}$ before and after surgery (Fig. 1). The results of this study are in accordance with a study stating that the patients switched from premix to Glargine and the addition of prandial insulin was associated with significant improvement in glycemia control [15]. A statistically significant difference $(p<0.05)$ existed preoperatively in mean before breakfast and before dinner blood glucose levels between the groups. The pre and postoperative blood glucose levels recorded in patients who were treated with Detemir+Aspart regime were comparatively lower than the patients who were treated with Glargine+Lispro regime. However, this difference was not statistically significant $(\mathrm{p}>0.05)$. These results are supported by the study reporting that the lower glucose levels were recorded in patients who were treated with insulin Detemir than the patients who were treated with NPH insulin [16]. Besides efficacy, safety of the treatment regime is equally important to reduce the morbidity and mortality. The diabetics undergoing surgery are more prone to adverse outcomes than non diabetics. This study has recorded $13.0 \%$ hypoglycemic episodes in patients who were treated with Glargine+Lispro regime and $15.1 \%$ hypoglycemic episodes in patients who were treated with Detemir+Aspart regime. However, this difference in percentage of hypoglycemic events between the groups was statistically insignificant ( $p>0.05$ ) (Fig. 2). Metabolic management and tight glycemia control during surgery has a significant impact on the risk of infectious complications including wound infection, urinary tract infection, and sepsis in patients with diabetes across a variety of surgical procedures [17]. Incidence of wound infection, urinary tract infection and gastrointestinal complications in addition to hypoglycemic events were also recorded in both the treatment regimens. $7.04 \%$ wound infections were recorded in patients who were treated with Detemir+Aspart regime and $11.59 \%$ wound infections in the patients who were treated with Glargine+Lispro regime, respectively. A significant difference existed in incidence of gastrointestinal complications $(\mathrm{p}<0.001)$ and urinary tract infections $(\mathrm{p}<0.05)$ between treatment groups. However, no statistically significant difference existed in other postoperative complications recorded between groups. Interestingly, the incidence of total number of complications 
recorded in patients who were treated with Detemir+Aspart regime were significantly lower $(\mathrm{p}<0.05)$ than the patients who were treated with Glargine+Lispro regime. The less percentage of complications recorded in patients who were treated with Detemir+Aspart regime could comparatively be due to lower mean blood glucose profile achieved during perioperative period. These findings are supported by a study reporting that maintaining the postoperative mean blood glucose levels less than $200 \mathrm{mg} / \mathrm{dl}$ during peri-operative period reduces the incidence of infection in diabetic patients undergoing surgery [18]. The mean daily insulin dose employed to achieve the glycemic target recorded in patients who were treated with Detemir+Aspart and Glargine+Lispro was $31.5 \pm 0.6$ units and $30.46 \pm 1.4$ units, respectively.

Both the treatment regimens can be used for glycemia control in diabetics undergoing surgery, however, insulin Detemir once daily and insulin Aspart before meals resulted comparatively in better clinical outcome. Simultaneous estimation and assessment of counter regulatory hormones and their control during intra-operative and post-operative period in diabetics undergoing surgery could further validate the evidence regarding the rational bases, as discussed, in defining the differences in the two treatments in the present study, however, in that direction resource constraint was the major limitation of this study. Therefore, more short and long term studies are warranted to investigate the significance and causal relationships of the differences in the outcomes with the treatments of split-mixed combination of long acting basal and rapid acting meal-time insulin regimes in larger number of diabetic population undergoing surgery.

\section{CONCLUSION}

The present study suggests that a significant difference may be existing in the postoperative clinical outcome in terms of glycemia control and complications between Detemir+Aspart and Glargine+Lispro insulin regimes in diabetic patients undergoing surgery. The insulin Detemir once daily and insulin Aspart before meals resulted comparatively in better glycemia control with minimum number of complications at low dose.

\section{CONFLICT OF INTEREST}

The authors confirm that this article content has no conflict of interest.

\section{ACKNOWLEDGEMENTS}

Declared none.

\section{REFERENCES}

[1] Umpierrez GE, Isaacs SD, Bazargan N. Hyperglycemia: an independent marker of in-hospital mortality in patients with undiagnosed diabetes. J Clin Endocrinol Metab 2002; 87(3): 97882.

[2] Shamoon H, Hendler R, Sherwin RS. Synergistic actions among anti insulin hormones in pathogenesis of stress hyperglycemia in humans. J Clin Endocrinol Metab 1981; 52(6): 1235-41.

[3] Jesson ME. Glucose control during cardiac surgery: how sweet it is. J Thorac Cardiovasc Surg 2003; 12(5): 985-7.

[4] Vasudev M, Jann M. Inpatient management of hyperglycemia and diabetes. Clin Diabetes 2011; 29: 5-9.

[5] Inzucchi SE. Management of hyperglycemia in hospital setting. N Engl J Med 2006; 355: 1903-11.

[6] Amiel SA, Alberti KGMM. Diabetes and Surgery. 6th ed. New York: McGraw Hill 2002.

[7] Hoogwerf BJ. Postoperative management of diabetic patient. Med Clin North Am 2001; 85: 1213-28.

[8] Samuel DJ. Management of diabetes mellitus in surgical patients. Diabetes Spectr 2002; 15: 44-8.

[9] Clement S, Braithwaite SS, Magee MF, et al. Management of diabetes and hyperglycemia in hospitals. Diabetes Care 2004; 27 : 553-91.

[10] Golden SH, Peart VC, Kao WH, Brancati FL. Peri-operative glycemic control and the risk of infectious complications in a cohort of adults with diabetes. Diabetes Care 1999; 22: 1408-14.

[11] Rosenberg CS. Wound healing in the patients of Diabetes Mellitus. Nurs Clin North Am 1990; 25: 247-61.

[12] Gottrup F, Andreassen TT. Healing of incisional wounds in stomach and duodenum: the influence of experimental diabetes. J Surg Res 1981; 31: 61-8.

[13] McMurry JF Jr. Wound healing and diabetes mellitus. Better glucose control for better wound healing in diabetes. Surg Clin North Am 1984; 64: 769-78.

[14] Furnary AP, Zerr KJ, Grunkemeir GL, Starr A. Continuous intravenous insulin infusion reduces the incidence of deep sternal wound infection in diabetic patients after cardiac surgical procedures. Ann Thorac Surg 1999; 67: 352-62.

[15] Davies M, Sinnasammy P, Storms F, Gomis R. Insulin glargine based therapy improves glycemic control in patients with type 2 diabetes sub-optimally controlled on pre-mixed insulin therapies. Diabetes Res Clin Pract 2008; 79: 368-75.

[16] Russell-Jones D, Simpson R, Hylleberg B, Draeger E, Bolinder J. Effects of insulin detemir or neutral protamine Hagedorn on blood glucose control in patients with type I diabetes mellitus using a basal-bolus regimen. Clin Ther 2004; 26: 724-36.

[17] Dronge AS, Perkal MF, Kancir S, et al. Long-term glycemic control and postoperative infectious complications. Arch Surg 2006; 141: 375-80.

[18] Zerr KJ, Furnary AP, Grunkemeier GL, et al. Glucose control lowers the risk of wound infection in diabetics after open heart operations. Ann Thorac Surg 1997; 63: 356-61.

Received: January 12, 2015
(C) Ahmad et al.; Licensee Bentham Open.

Revised: February 22, 2015

Accepted: February 28, 2015

This is an open access article licensed under the terms of the Creative Commons Attribution Non-Commercial License (http://creativecommons.org/licenses/ by-nc/3.0/) which permits unrestricted, non-commercial use, distribution and reproduction in any medium, provided the work is properly cited. 